2 Day S, Ward H, Harris JRW. Prostitute women and public health. $B M \mathcal{F}$ 1988;297:1585

3 Tirelli U, Rezza G, Guiliani M, Caprilli F, Gentilli G, Lazzarin A, et al. HIV seroprevalence among 304 female prostitutes from four Italian towns. AIDS 1989;3:547-8

4 Parry J, Perry K, Mortimer P. Sensitive assays for viral antibodies in saliva: an alternative to tests on serum. Lancet 1987;ii:72-5.

5 Tappin DM, Girdwood WA, Follet EAC, Kennedy R, Brown AJ, Cockburn F. Prevalence of maternal infection in Scotland using an unlinked anonymous testing of newborn babies. Lancet 1991;337:1565-7.

6 LePorte RE, McCarty DJ, Tull ES, Tajima N. Counting birds, bees, and NCDs. Lancet 1992;339:494-5.

7 Hook EB, Regal RR. Capture-recapture methods. Lancet 1992;339:742.

8 Frischer $M$, Leyland AH. Reliability of population and prevalence estimates. Lancet 1992;339:995.

9 McKeganey NP, Barnard MA, Bloor MJ, Leyland AH. Injecting drug use and female streetworking prostitution in Glasgow. AIDS 1990;4:1153-5.

10 Bloor MJ, Leyland AH, Barnard MA, McKeganey NP. Estimating hidden populations: a new method of calculating the prevalence of drug injecting and non-injecting female prostitutes. Br f Addiction 1991:86:1477-83.
11 Cormack RM Log-linear models for capture-recapture. Biometrics 1989:45: $395-413$

12 Leyland AH, Barnard M, McKeganey N. The use of capture recaptur methodology to estimate and describe covert populations: an application to female streetworking prostitution in Glasgow. Paper presented to the International Conference on Social Science Methodology, University of Trento, Italy, 22-26 June 1992.

$13 \mathrm{Kinnell} \mathrm{H.} \mathrm{Prostitutes,} \mathrm{their} \mathrm{clients} \mathrm{and} \mathrm{risks} \mathrm{of} \mathrm{HIV} \mathrm{infection} \mathrm{in} \mathrm{Birmingham}$ Birmingham: Dept of Public Health Medicine, 1989. (Occasional paper.)

14 Morgan Thomas R, Plant MA, Plant MC, Sales DI. Risks of AIDS amon workers in the sex industry: Some initial results from a Scottish study. $B M \mathcal{F}$ 1989;299:148-9.

15 McKeganey NP, Barnard MA. Selling sex: female street prostitution and HIV related risk behaviour in Glasgow. AIDS Care (in press).

16 Frischer M, Bloor M, Finlay A, Goldberg D, Green S, Haw S, et al. A new method of estimating the prevalence of injecting drug use in an urban

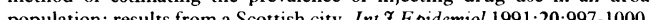

(Accepted 3 September 1992)

\title{
Computer system for assisting with clinical interpretation of tumour marker data
}

\author{
M S Leaning, S Gallivan, E S Newlands, J Dent, M Brampton, D B Smith, K D Bagshawe
}

\section{Abstract}

Objective-To design and evaluate a computer advisory system for the treatment of gestational trophoblastic tumour.

Design-A comparison of clinicians' treatment decisions with those of the computer system. Two datasets were used: one to calibrate the system and one to independently evaluate it.

Setting-Department of medical oncology.

Patients-Computerised records of 290 patients with low risk gestational trophoblastic tumour for whom the advisory system could predict the adequacy of treatment. The calibration set comprised patients admitted during 1979-86 (227) and the test set patients during 1986-89(63).

Main outcome measures-The system's accuracy in predicting need to change treatment compared with clinicians' actions. The mean time faster that the system was in predicting the need to change treatment.

Results -On the calibration dataset the system was $94 \%(164 / 174)$ accurate in predicting patients whose treatment was adequate, recommending change when none occurred in only $10(6 \%)$ patients. In patients whose treatment was changed the system recommended change earlier than clinicians in 39/53 cases $(74 \%)$, with a mean time advantage of 14.9 (SE $2 \cdot 02$ ) days. On the test dataset the system had an accuracy of $91 \%(31 / 34)$ in predicting treatment adequacy and a false positive rate of $9 \%(3 / 34)$. The system recommended change earlier than clinicians in $22 / 29$ cases $(76 \%)$, with a mean time advantage of $12 \cdot 5(2 \cdot 22)$ days.

Conclusions-The computer advisory system could improve patient management by reducing the time spent receiving ineffective treatment. This has implications for both patient time and clinical costs. Oncology, Charing Cross Hospital, London W6 8RF E S Newlands, professor of cancer medicine

J Dent, research officer

M Brampton, data manager

D B Smith, senior registrar

K D Bagshawe, emeritus

professor of oncology

Correspondence to:

Dr Leaning.

BM 1992;305:804-7

Introduction
Serial measurements of serum human chorionic
gonadotrophin are central to the management of
patients with gestational trophoblastic tumour. ${ }^{1}$ Serum
human chorionic gonadotrophin concentration is
monitored before, during, and after cytotoxic chemo-
therapy to assess tumour growth and the response to
treatment. During successful treatment, human
chorionic gonadotrophin concentrations decrease

Introduction
Serial measurements of serum human chorionic
gonadotrophin are central to the management of
patients with gestational trophoblastic tumour. ${ }^{1}$ Serum
human chorionic gonadotrophin concentration is
monitored before, during, and after cytotoxic chemo-
therapy to assess tumour growth and the response to
treatment. During successful treatment, human
chorionic gonadotrophin concentrations decrease
Introduction
Serial measurements of serum human chorionic
gonadotrophin are central to the management of
patients with gestational trophoblastic tumour. Serum
human chorionic gonadotrophin concentration is
monitored before, during, and after cytotoxic chemo-
therapy to assess tumour growth and the response to
treatment. During successful treatment, human
chorionic gonadotrophin concentrations decrease rapidly. If, however, treatment becomes ineffective the concentration will fall more slowly, plateau, or rise. It is important to detect drug resistance as soon as possible and to modify the patient's treatment appropriately.

In a preliminary analysis we examined human chorionic gonadotrophin concentration during treatment in $\mathbf{3 4 0}$ low risk patients with gestational trophoblastic tumour attending the department of medical oncology at Charing Cross Hospital during 1979-86. Two groups of patients were distinguished: those who responded successfully to one treatment and those who needed to change treatment because of drug resistance (two treatment group). Patients whose treatment was changed because of toxicity were excluded. Mean initial human chorionic gonadotrophin concentrations at the start of treatment and mean initial regression slopes were significantly different $(p<0.002)$ for the two groups, but this difference was not sufficient to discriminate accurately between the two groups. A linear discriminant analysis using initial human chorionic gonadotrophin concentration and initial regression slope as factors produced a $33.9 \%$ false positive rate (one treatment patients assigned to the two treatment group) and a $31 \cdot 7 \%$ false negative rate (two treatment patients assigned to the one treatment group). We concluded that continuous monitoring of serum human chorionic gonadotrophin was necessary during treatment.

Even with regular measurements of human chorionic gonadotrophin during treatment interpreting the marker response can be difficult, despite routine graphical display of the data on a computer system. This paper describes the design and evaluation of a computer advisory system (MARKER) intended to improve the management of low risk gestational trophoblastic tumour. The aim of this system is to allow a clinician to detect an inadequate treatment response as soon as possible. This is done by forecasting the trend of the human chorionic gonadotrophin concentration and comparing the predicted time to basal concentration $(<5 \mathrm{IU} / \mathrm{l})$ with an expected distribution of times derived from a database of past cases.

This approach may also be applicable to other cancers with serum markers, in particular to germ cell tumours and ovarian cancer. The general principle underlying the approach to computer assisted treatment monitoring is that a formal algorithm is used to 
forecast responses continually and compare them with the expected response for the individual patient. We expect that this principle will be widely applicable.

\section{Methods}

DESIGN OF PROGRAM

The tumour marker advisory program (MARKER) is written in Turbo Pascal and runs in MS DOS on IBM and compatible personal computers. Its primary functions are to act as a database system for tumour marker data; to display time series data graphically; to compute and display best fit straight lines to highlighted portions of a time series and to forecast the trend; and, to display distributions of expected time to basal concentration, based on a calibration dataset of low risk patients.

MARKER is intended to augment and not replace clinical decision making. Clinicians use the graphical information from the program (expected times to basal concentrations) and other clinical data to evaluate and modify treatment.

Each patient record consisted of a patient identification number, the date of start of treatment, the date of change of treatment (if any), the date of final treatment, an indicator showing whether treatment was changed because of toxicity (this group was excluded from the analysis), and an indicator showing whether treatment changed because of failure to respond to the initial therapy. In addition, a patient's record contained a sequence of serum human chorionic gonadotrophin concentrations and their corresponding dates. In most cases these values covered periods before the start of treatment and extended beyond the end of treatment. Most data analysed began at the start of treatment, but if no value was recorded for the first day of treatment the value preceding treatment was taken.

MARKER can display the reference distribution of expected times to basal concentrations and best fit lines along with the tumour marker data. Figure 1 shows the screen with both displayed shortly after the start of treatment. Preliminary analysis showed that for the one treatment group cure time (when human chorionic gonadotrophin concentration falls below a basal value of $5 \mathrm{IU} / \mathrm{l}$ ) was related to the logarithm (base 10) of human chorionic gonadotrophin concentration at the start of treatment. The reference distributions were based on a regression analysis of the data obtained from 1979 to 1986 (fig 2). The basal concentration is the upper limit of the normal range for the human chorionic gonadotrophin assay.

The patient data to be fitted are indicated on the screen with square brackets (fig 1), which can be moved by using the arrow keys on the keyboard. The

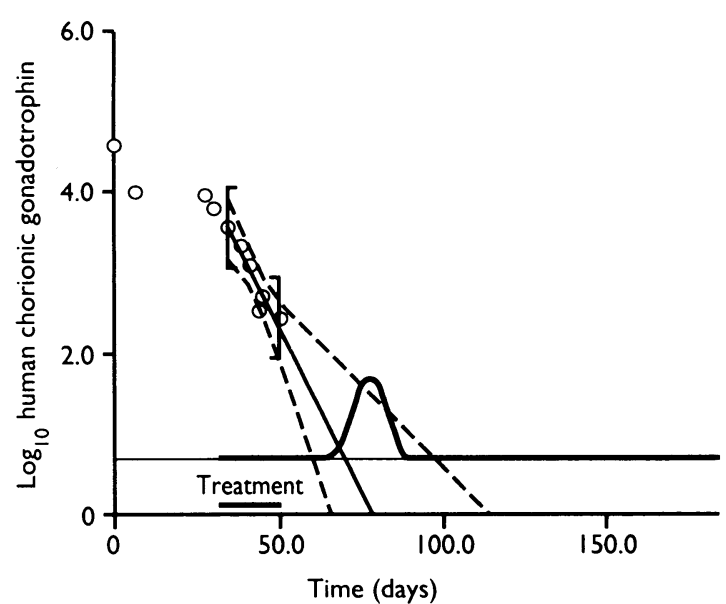

FIG 1-Display of patient human chorionic gonadotrophin data for one patient in advisory program MARKER. Forecast time to basal values is within reference probability distribution

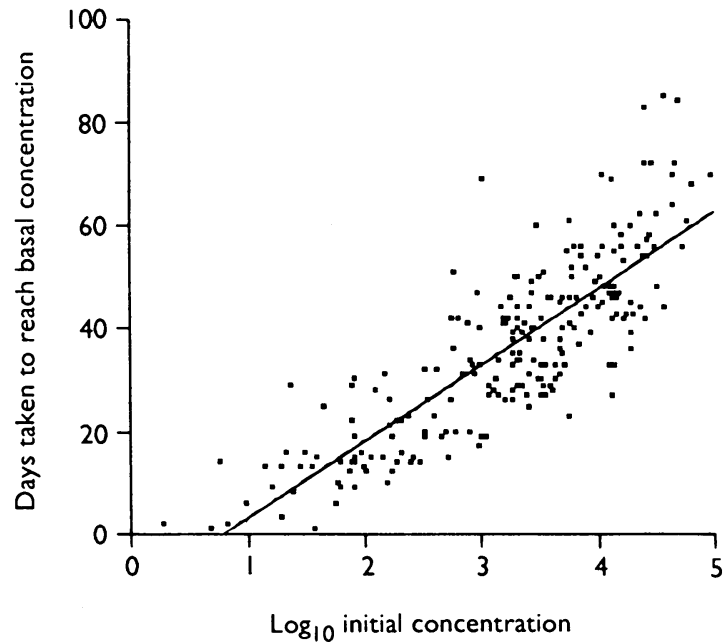

FIG 2-Relationship between time taken for patients to reach basal human chorionic gonadotrophin values and initial serum human chorionic gonadotrophin concentrations

best fit line and prediction appear as a solid line and their $95 \%$ confidence limits as dotted lines. These lines are derived with the standard equations of linear regression $^{3}$ expressed in recursive form for efficiency. In figure 1 the projected tumour marker decay predicts a time to basal levels within the low risk distribution so treatment would not need to be changed. Although the prediction is an extrapolation of the regression line, it should be interpreted as what would happen if the patient continued to respond at this rate. Figure 3 shows the same patient nine days later. The brackets

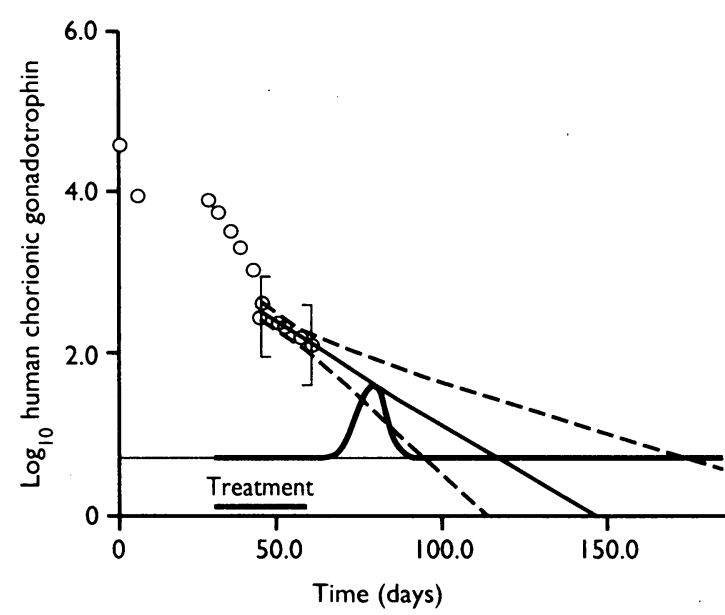

FIG 3-Data for same patient as in figure 1 nine days later. The forecast is at an extreme end of the probability distribution, suggesting treatment change may be necessary

have been set to forecast the trend of the last six data points. The predicted time to basal concentration is on the extreme upper tail of the low risk distribution. A clinician would probably interpret this as the need to change treatment.

The evaluation results reported later suggest that six is a reasonable and effective number of data points to include, allowing slope changes (and potential drug resistance) to be detected earlier than without the system. It is desirable, therefore, to fix the regression interval to six points when the system is in clinical use. Until further evaluation has been done of using other numbers of data points, varying this number might lead to misleading conclusions.

\section{EVALUATING THE SYSTEM}

Many criteria should be used to evaluate a clinical computer system including ease of use, accessibility, cost, and flexibility. The criterion that will be discussed here is the quality of the decision support that 
the system provides. This is difficult to quantify since the system has been designed to help decision making rather than substitute for the clinician. Little data are available about the quality of decisions made by clinicians assisted by the system compared with decisions made without its aid.

To quantify some aspects of the system's performance, a separate computer program was written to mimic the operation of the system and the treatment recommendations (a feature specifically avoided in the design of the main system). This "surrogate advisory system" was used to examine retrospective patient data transferred from the clinical database at Charing Cross Hospital. Treatment recommendations made by the surrogate advisory system were compared with the actual decisions of medical oncologists. The surrogate advisory system automated the application of the treatment recommendation rule to a group of patient records, unlike the main advisory system which is designed to help clinicians to manage individual cases.

The data transferred from the Charing Cross clinical database concerned all low risk patients with gestational trophoblastic tumour referred between 1979 and 1989. The total number of patient records transferred was 430. Patient records were transferred in two groups. The first group (the calibration group) corresponds to patients referred during 1979-86. These data were used both for the analysis that gave rise to the original system design and for calibrating some of the parameters used by the system. The second group (the test group), patients referred during 1986-89, was used purely for evaluating the system. Some patients' records were excluded for the following reasons: serum human chorionic gonadotrophin concentration before treatment was unavailable, insufficient data points to carry out regression analysis before basal concentration of human chorionic gonadotrophin were achieved, basal concentrations of human chorionic gonadotrophin $(5 \mathrm{IU} / \mathrm{l})$ never attained, insufficient data points to carry out regression analysis before treatment was changed, insufficient human chorionic gonadotrophin concentrations recorded above basal concentration to carry out regression analysis, treatment time exceeded one year.

The main advisory system could have been used for such patient records, but using its regression facilities would have been inappropriate. In addition, patients whose treatment was changed because of toxicity were also excluded from the analysis as the advisory system is designed only to assess response and non-response to treatment.

For the purposes of the evaluation six data points for human chorionic gonadotrophin hormone were used in assessing trends. The system scans through each successive value after the start of treatment fitting a linear function of time to the logarithms of the last six values by linear regression. This function is extrapolated until it reaches the basal concentration of 5 IU/l (as in figure 1). The surrogate system recommends treatment change if the forecast time to reach basal concentration exceeds the maximum allowable time, which is a function of the initial human chorionic gonadotrophin concentration (fig 2): maximum time to basal concentration (days) $=-12 \cdot 0+14 \cdot 9 \log _{10}$ (initial human chorionic gonadotrophin $+\mathrm{t}$. $S$, where $S=9 \cdot 49$ is the standard deviation of the residuals about the regression line and $t$ is a factor adjusted during the calibration phase.

The surrogate advisory system reported patients for whom data were incompatible with operating the program. For those with compatible data it reported whether a change in treatment would be recommended for a given patient and the date on which such a recommendation would be made. This information was compared with the clinicians' decisions.
A decision to change treatment was defined as a positive decision. Thus assignment of one treatment patients by the advisory system to the two treatment group was described as a false positive decision. A false negative decision was assignment of two treatment patients to the one treatment group. False positive decisions imply patients receiving a change of treatment that they may not require and false negative decisions that treatment is changed before the advisory system has suggested it. True negative decisions are those when the system advises change before the clinician. For this subgroup of patients the mean time benefit of the system was calculated.

The aim of the calibration phase was to minimise the false positive rate while maximising the mean time benefit. The calibration was performed by adjusting the calibration factor $t$ in the above equation. We hoped to achieve a false positive rate less than $10 \%$ and a time benefit of at least two weeks in the two treatment group. Once best performance had been obtained on the calibration set, the surrogate advisory system was evaluated on the test set.

\section{Results}

After exclusion of patients whose treatment was changed because of toxicity and those whose data were incompatible with operation of the surrogate advisory system 227 patients remained in the calibration set and 63 patients in the test set. The quality of the decisions a clinician would have made aided by the main system for the excluded patients cannot be assessed.

The remaining patients can be divided into two classes: those for whom a treatment change was actually recommended (two treatment group); and those who completed treatment without requiring a change to more aggressive therapy (one treatment group).

The treatment recommendations made by the surrogate advisory system (SAS) for the calibration data set were obtained with the calibration factor set to $4 \cdot 0$. The same calibration factor was used in evluating the test set. The table summarises the treatment recommendations of the surrogate advisory system for the calibration and test sets. For the calibration set $(227$ patients) 174 completed chemotherapy without changing treatment. The advisory system advised change in 10 of these 174, a $6 \%$ false positive rate. Fifty three patients required a change of chemotherapy because of drug resistance. In 14 cases the clinicians changed the treatment before the advisory system recommended it, a $26 \%$ false negative rate, while in 39 cases the system advised change before the clinicians (74\%). For these 39 patients the mean time to change was 14.9 (SE 2.02) days earlier with the advisory system than with the clinicians. Thirty four of the 63 patients in the test dataset completed treatment without changing. In three of these the system advised change, a $9 \%$ false positive rate. Twenty nine patients changed chemotherapy because of drug resistance, and in seven the

Assignment of patients (percentages in parentheses) in the calibration and test datasets to one or two treatment groups by surrogate advisory system ( ${ }^{\star}$ treatment changed by clinicians before computer, tcomputer advised change incorrectly, ftreatment changed by computer first)

\begin{tabular}{lcc}
\hline \multirow{2}{*}{$\begin{array}{l}\text { Surrogate advisory } \\
\text { system's decision }\end{array}$} & \multicolumn{2}{c}{ Clinicians' decision } \\
\cline { 2 - 3 } & $\begin{array}{c}\text { 1 Treatment } \\
\text { (no change) }\end{array}$ & $\begin{array}{c}\text { 2 Treatments } \\
\text { (change) }\end{array}$ \\
\hline Calibration set & \\
1 Treatment & $164(94)$ & $14(26)^{\star}$ \\
& $10(6) \dagger$ & $39(74) \ddagger$ \\
Treatments & Test set & \\
2 Treatment & $31(91)$ & $7(24)^{\star}$ \\
\hline
\end{tabular}


clinicians changed before the system, a $24 \%$ false negative rate. The mean time saving for the 22 patients in whom the system advised change first was 12.5 (SE $2 \cdot 22$ ) days.

\section{Discussion}

This study shows that in gestational trophoblastic disease a computer advisory system would allow $75 \%$ of patients who need to change chemotherapy because of drug resistance to do so an average of 12-14 days earlier than would be the case when relying solely on clinical judgment. In addition, the system had an acceptably low false positive rate-that is advised unnecessary change in therapy-of $5-8 \%$. This represents a group of patients whose human chorionic gonadotrophin concentrations fall slowly but progressively and whose half life for the hormone is outside the range accepted by the system. In practice these patients might be recognised by clinical staff, thus further reducing the false positive rate and increasing the savings in patient treatment times and drug costs.

\section{SERUM TUMOUR MARKERS}

The prognostic value of half lives of serum tumour markers has been investigated in several other malig nancies. In 1982 Lange $e t$ al divided a series of patients with testicular germ cell tumour into good and poor prognosis groups on the basis of human chorionic gonadotrophin and $\alpha$ fetoprotein half life. ${ }^{4}$ One of 10 patients with a half life for human chorionic gonadotrophin above three days or for $\alpha$ fetoprotein above six days, or both, achieved complete remission compared with 12 of 19 with normal marker half lives. They attempted to validate this formula in a subsequent group of patients but found no difference in the $\alpha$ fetoprotein half life for patients achieving complete remission and those who did not. ${ }^{5}$

Using a similar approach Picozzi et al reported the separation of patients with germ cell tumour into high and low risk groups on the basis of day 1 and day 22 human chorionic gonadotrophin and $\alpha$ fetoprotein values. ${ }^{67}$ The rate of complete remission was $51 \%$ in the low risk group and $92 \%$ in the high risk group $(\mathrm{p}<0.001)$; multivariate analysis found that other prognostic factors did not improve prediction of outcome. However, when the European Organisation for Research and Treatment of Cancer genitourinary group attempted to validate this model in patients treated in three successive studies they found rates for incorrect prediction of treatment failure of $40 \%, 58 \%$, and $83 \%$ respectively. ${ }^{8}$ It was concluded that the model was not reproducible.

More recently Toner $e t a l$ have looked at the half lives of $\alpha$ fetoprotein and human chorionic gonadotrophin in 198 patients with germ cell tumours using the first two samples taken between day 7 and day 90 after the start of chemotherapy. ${ }^{9}$ With abnormal half life defined as above seven days for $\alpha$ fetoprotein and above three days for human chorionic gonadotrophin 42 patients had a prolonged half life for either $\alpha$ fetoprotein or human chorionic gonadotrophin. Of these 42 patients, $12(29 \%)$ achieved complete remission compared with 139 of $156(89 \%)$ patients with normal marker half lives $(p<0 \cdot 0001)$. Moreover eight of 12 patients with prolonged half lives relapsed $(67 \%)$ compared with 18 of $139(13 \%)$ of those with a normal half life. Cox multivariate analysis showed that prolonged marker half life was the single most important factor for the prediction of tumour free survival in this group of patients.
The relation of CA 125 half life with prognosis in ovarian cancer after starting chemotherapy has been investigated by several groups. Three studies found that those patients with a half life for CA 125 above 20 days had a worse prognosis than those with a half life below 20 days..$^{10-13}$ In addition Rustin $e t$ al showed that eight of $14(58 \%)$ patients with a greater than sevenfold fall in CA 125 concentration 28 days after starting chemotherapy remained disease free compared with three of $36(9 \%)$ with a smaller fall $(p=0 \cdot 0005)$. This formula is currently being validated on a different dataset by the Medical Research Council.

\section{EXTENDING THE SYSTEM}

These studies all suggest that assessing tumour marker half life during treatment gives important prognostic information. However, none of the formulas derived has been successfully validated. Moreover, the considerable population overlap in all the situations studied make predictions on an individual patient basis difficult. Our method seems to overcome this problem. The results show that continuous estimation of the rate of fall of human chorionic gonadotrophin concentration rather than concentrating on the initial period after starting treatment allows the outcome of treatment to be accurately predicted with a low false positive rate. The method has been successfully validated with a separate dataset. We intend to incorporate the computer advisory system into the routine management of patients with gestational trophoblastic tumour and propose to extend the investigation of this approach to patients with medium and high risk gestational trophoblastic tumour and then to germ cell tumours and possibly ovarian cancer.

The Clinical Operational Research Unit is supported in part by the Department of Health. We thank Professor Ray Jackson for his encouragement and advice. This work was supported in part by the Cancer Research Campaign.

1 Bagshawe KD, Dent J, Newlands ES, Begent RHJ, Rustin GJS. The role of low dose methotrexate and folinic acid in gestational trophoblastic tumours (GTT). B F Obst Gynaecol 1989;96:795-802.

2 Bagshawe KD. Risk and prognostic factors in trophoblastic neoplasia. Cancer 1976;38:1373-85.

3 Armitage P. Statistical methods in medical research. 2nd ed. Oxford: Blackwell, 1987.

4 Lange H, Vogelzang N, Goldman A, Kennedy B, Fraley E. Marker half-life analysis as a prognostic tool in testicular cancer. $\mathcal{F}$ Urol 1982;128:708-11.

5 Vogelzang N, Lange P, Goldman A, Vessels R, Fraley E, Kennedy B. Acute changes of alfa fetoprotein and human chorionic gonadotrophin during induction chemotherapy of germ cell tumours. Cancer Res 1982;42:4855-61.

6 Picozzi V, Hannigan J, Freina F, Torti F. Prognostic significance of a decline in serum human chorionic gonadotrophin levels after initial chemotherapy for advanced germ-cell carcinoma. Ann Intern Med 1984;100:183-6.

7 Picozzi V, Hannigan J, Freina F, Rogoway W, Torti F. Prognostic significance of day 1 and day 22 marker levels in advanced male germ cell cancer. Proceedings of the American Society of Clinical Oncology 1985;910:102.

8 Bartels C, Stoter G, Kay S. Prognostic significance of day 22/day 1 ratio of tumour marker levels in disseminated nonseminomatous testicular cancer. Proceedings of ECCO 1987:179.

9 Toner G, Geller L, Tan C, Nisselbaum J, Bosi G. Serum tumour marker halflife during chemotherapy allows early prediction of complete response and life during chemotherapy allows early prediction of complete response and
survival in nonseminomatous germ cell tumours. Cancer Res 1990;50: 5904-1.

10 van der Burg M, Lammes F, van Putten W, Stoter G. Ovarian cancer: the prognostic value of the serum half-life of CA125 during induction chemotherapy. Gynecol Oncol 1988;30:307-12.

11 Hawkins R, Roberts K, Wiltshaw E, Mundy J, Fryatt I, McCready V. The prognostic significance of the half-life of serum CA125 in patients responding to chemotherapy for epithelial ovarian carcinoma. Br $\mathcal{O}$ Obstet Gynaecol 1989;96:1395-9.

12 Hunter V, Daly L, PA-CM, Soper JT, Berchuk A, Clarke-Pearson L, et al. The prognostic significance of $\mathrm{CA} 125$ half-life in patients with ovarian cancer who have received primary chemotherapy after surgical cytoreduction. Am F Obstet Gynecol 1990;163:1164-7.

13 Rustin G, Gennings J, Nelstrop A, Covarrubias H, Lambert H, Bagshawe K. Use of CA-125 to predict survival of patients with ovarian carcinoma. $7 \mathrm{Clin}$ Oncol 1989;7:1667-71.

(Accepted 30 fuly 1992) 